\title{
Environment versus man radioprotection: The need for a new conceptual approach?
}

\author{
F. Bréchignac \\ Institute of Protection and Nuclear Safety, Department for Environmental Protection, \\ Division for Laboratory Radioecological Research and Studies, Centre de Cadarache, \\ BP. 1, 13108 Saint-Paul-lez-Durance cedex, France
}

\begin{abstract}
There is a present consensus in that environment radioprotection is essentially to deal with chronic multiple exposures to low dose rates. In terms of protection, man and the environment are not similar in nature in that the environment is not equivalent to a single species community to be protected. The immediate question which arises therefore is: what do we want to protect, or what do we mean by the environment? It is proposed that the keyword here is the "ecosystem", i.e. a complex-organized self-sustaining living entity to which man survival is fully subordinated. This leads to a new conceptual approach which also evolves from the growing public "feeling" (not yet appropriately substantiated in scientific terms, however) that if the environment is properly protected, then man would not be harmed. Along this line, the position of man with respect to the environment is to be reconsidered. As an inherent part of it, he is both capable of promoting substantial change of its structure and function, but also remains fully subordinated to the life support function that it hosts. In line with this conceprual view, this paper suggests an innovative approach designed around closed artificial ecosystems, as simplified reference models prone to support experimental investigations on chronic low-dose effects in controlled conditions.
\end{abstract}

\section{INTRODUCTION}

Radioprotection has first, and certainly with full justification, dealt with protection of man. The philosophy, the concepts and the practical application of this protection have been elaborated through an international consensus by the ICRP. Until recently, the concern about protecting the environment from ionising radiations has therefore been conceptualised with the essential goal of protecting human beings. In this context, the environment has been taken into account in a restrictive manner, i.e. as a potential source of harm to human health via the food chain, without considering the potential harm to the nonhuman biota themselves. This was justified from an analysis of the vast literature on biological effects induced by ionising radiation which indicated that mammalians, and particularly humans, were most radiosensitive among the life kingdom, a feature which led ICRP to state that the current standards used for protecting humans would also provide, in an implicit manner, an acceptable protection of non-human biota, at least for their populations if not their individuals $[1,2]$.

However, emerging from recent concepts in environmental protection such as "sustainable development" and the "precautionary principle", a worldwide debate is currently growing towards a less anthropocentric consideration of the environment and its biota. This has boosted the search for better understanding of key planetary issues such as biodiversity, global climate change, environmental toxicology, and also now, as a logical consequence, environment radioprotection, asking for a reevaluation of the validity of the ICRP statement. Indeed, the scientific background from which evolved this statement is essentially restricted to short-term effects on individuals in acute exposure situations (high doses) whereas the most general concern in the environment roots from long-term effects (potential harm to future generations) in situations of diffuse contaminations promoting low dose exposure of populations, which are further structured temporally and spatially through complex interactions referted to as ecosystems. The existing scientific background therefore still largely misses the real issue.

Furthermore, an often-cited weakness of the ICRP statement due to its evolvement from man protection mentions that biota located in environments not directly in interaction with humans may not be adequately protected [3]. This has been highlighted for example from the previous practice of radioactive waste dumping in deep seas where radiation levels to the local biota could be quite high without endangering men. 


\section{WHY AND WHAT IS TO BE PROTECTED?}

These questions seem trivial, but the answers are in fact often poorly defined leading to a variety of understanding which puzzle an efficient and coherent advancement of the worldwide growing scientific effort.

First of all, it must be recognised that in terms of protection, man and the environment are distinct entities placed at different levels of organisation. The environment, and in particular its biota, cannot be reduced to a unique species forming a single population of individuals. It results from this that both radioprotection systems, for humans and the environment, cannot be identical, but they still need to remain compatible one with the other.

Second, one basement of the planetary environmental concern roots from the emerging consciousness that man is an inherent part of the biosphere, and that this later hosts and supports processes which are vital for the survival of humankind, therefore deserving attention and protection. This means a necessary move from an anthropocentric approach - man as endpoint - to a more biocentric approach - man within the environment, as a piece of a larger system including non-human biota (Figure 1). At variance from human radioprotection based on a linear concept, from a source towards man through the environment, radioprotection of the environment necessitates a "system" approach which would attempt to take into account the numerous, complex and cyclic interactions (the whole biosphere is closed) occurring between the physical environment (biotope) and the living populations (biocenose). This view drives to the "ecosystem" emerging as the ultimate endpoint of environmental radioprotection. It is the basic functional unit within the biosphere which, by assembling complex spatially and temporally structured interactions between the physical and living world, hosts the vital life support functions. In the global terrestrial ecosystem - the biosphere - one understands that man has become an actor with a quite significant impact, but meanwhile, his survival (as an inherent piece of the system) remains unavoidably subordinated to the fundamental laws which maintain some planetary homeostasy (temperature, breathable oxygen concentration, water and carbon cycling).

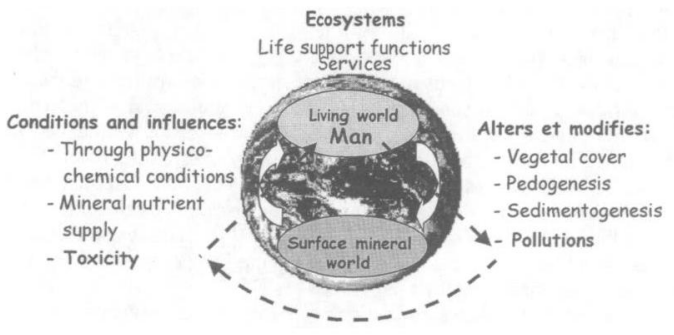

Figure 1. Biocentric approach featuring ecosystem functions as a key to environment protection.

\section{HOW TACKLING THE QUESTION?}

\subsection{The two complementary lines of approach}

There are two levels behind this question: 1) acquiring the appropriate scientific knowledge currently missing, and 2) deriving from this knowledge a consensual and practical system for environmental radioprotection. In response to a growing current social demand, there is a large brain storming today aimed at elaborating a coherent framework for radioprotection of the environment to be based on the current scientific knowledge available, but re-examined in a more structured and complete manner than previously (the FASSET European project, [4]). As the ICRP success has demonstrated for man radioprotection, it is indeed necessary not to wait for a full scientific understanding before setting up practical standards and references. The system will therefore need to evolve together with the 
improvement of the scientific knowledge. It must be recalled however that the present knowledge is not fully appropriate (as mentioned above), and that few innovative approaches to fill the gaps are currently proposed.

\subsection{The gaps in scientific knowledge}

Scientists from the american DOE, who have been first to propose dose standards for biota radioprotection, strengthened the weaknesses of this early approach: "while we believe these limits to be a reasonable assumption based on current knowledge (essentially on individuals), our full understanding of the ecological and evolutionary significance of molecular changes and of potential synergistic effects is too weak to ignore" [5]. The scientific basis for a well mastered understanding of the effects of radiations on biota at the higher levels of organisation is not yet acquired. Located at one end of the complexity scale of living entities, ecosystems are complex integrators, and the way they cope with toxicants introduced within their looped processes of energy and matter transformation is poorly understood. In addition, real situations of environment contamination usually consist of the concomitant occurrence of multiple pollutants, which have been recently demonstrated to yield synergistic and antagonistic effects, a feature which can no longer be ignored $[6,7,8]$.

It is of importance to highlight the quasi-ignorance of effects which may appear in the long-term, i.e. those which, farther than the scale of individuals may, through their reproduction, appear later on at the scale of populations. If reproduction-related criteria are recognised as being the most radio-sensitive, one must bear in mind that genetic damages are promoted at even lower dose rates, and that the long-term implications of such an observation is not known. For example, the vole populations surrounding Chernobyl do not appear to be suffering although they are exhibiting a clear alteration of the mitochondrial DNA coding for cytochrome b [9]. One can question if doses too weak to affect reproduction could nevertheless promote in the long-term a mutational pool in the population sufficient to alter the course of its evolution.

Another major lack of knowledge concerns the dose-effect relationship at the populations and ecosystems scale. A few studies driven in situ on contaminated natural environments have been reported recently, but the strength of their conclusions is suffering from the lack of controls inherent to this descriptive approach. A further major gap roots from our poor understanding of long-term effects of chronic exposures (several generations) at low dose rates.

\subsection{The difficulty of a holistic approach of the ecosystem}

In environmental radioprotection, the often-cited rationale for the current focus of research on individuals comes from the assumption that no effect can happen at the ecosystem level if this is not driven by some effects at the individual level. This may not be so simple as complex systems theory is now suggesting in view of better understanding ecosystem integrity [10]. This theory, evolving from non-equilibrium thermodynamics, considers ecosystems as highly hierarchical self-organising entities which proceed by capturing more resources, optimising the effective use of the resources, elaborating more structure and enhancing survivability. Such systems exhibit emergent dynamic behaviours which may turn out into abrupt discontinuities, with sudden changes. This is because they can persist only within an energetic window where they get sufficient energy, but not too much. Below a lower threshold, self-organisation simply cannot occur, and above another higher threshold, the organised structures fall apart as the energy overrides their dissipative ability. Upon any stress, like radiation, it is probable that the ecosystem response starts off with the initial alteration of the most sensitive components (which may not be trivial to detect), followed by the setting up of repair mechanisms, ultimately yielding either a new balance status (homeostasy restoration) or a fatal divergence. This highlights that proper evaluation of radiation effects at ecosystem level cannot simply be derived from effects observed at the level of individuals.

Even at population level, addressing radiotoxicity to biota through single-species tests may not be sufficient. This approach aims at measuring "direct effects" such as reduced survival, growth, reproduction or other attributes of exposed organisms. But this may be overrided by "indirect effects" driven by higher level processes at work within natural ecosystems, such as predation and competition, 
and ultimately yield erroneous interpretation of the results. A good illustration of a counter-intuitive environmental response has been shown regarding ultraviolet-B radiation: short-term, single-species, tests demonstrated reduced photosynthesis and growth of benthic diatoms population, but long-term, multi-population level, tests demonstrated an increased standing crop of diatoms because the radiation also inhibited chironomids larvae, which are algal consumers [11]. Predation and competition are known ecosystem-level processes which, through balancing inter-population relationships, act on ecosystem structure and resilience.

\section{USING CONTROLLED ECOLOGICAL MODELS}

\subsection{Generation of a scientific background suitable to support environmental assessment}

A quite original avenue to cope with the above-mentioned difficulties while filling the gaps in scientific knowledge (unravelling effects of chronic low doses of radiation at ecosystem level) is brought about by using controlled ecological models. This innovative approach has already been used in modern ecology with successful achievements by bringing new and powerful tools to issues such as biodiversity [12-13], climate change [14], and more recently to ecotoxicology [15-16-17] and even ecosystem radiotoxicology [18-19]. Laboratory ecosystem models fulfil a bridge between theory and nature. They are not a substitute for real ecosystems occurring in nature, but by simplifying its complexities, they sharpen our understanding of natural processes and give access to an appropriate formulation of the most pertinent new questions.

Laboratory ecosystem models do not intend to replace descriptive investigations in the real environment which will always be required, but as a further contribution to answering the same questions through another angle, their advantages nicely fill the shortcomings of the other approach, because they are replicable, reproducible, prone to mastering and manipulating environmental variables, and still hosting an ecosystem-representative level of complexity.

\subsection{What are controlled ecological models: various examples}

Basically, these are closed artificial ecosystems, reconstructed in the laboratory as model entities hosting natural ecosystem-level processes occurring in nature. This cannot be done without respecting the three basic functional biotic compartments, namely producers, consumers and decomposers, which, by virtue of their interactions (Figure 2), promote the life self-support inherent to all viable ecosystems. Indeed, simply enclosing a small portion of nature will never work because breaking out this vital functional structure. It is the need for simplification which drives to "artificial". This is the key to mastering environmental and biotic variables, quantifying the energy and matter transfer fluxes, and therefore understanding effects resulting from a toxicant-induced perturbation.

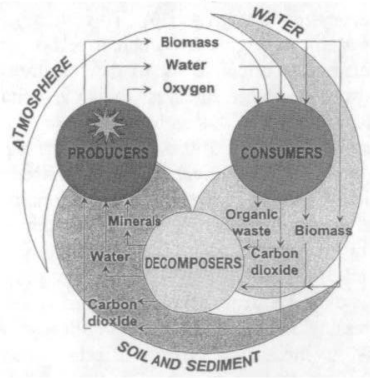

Figure 2. Schematic diagram of the basic life support functions within ecosystems featuring interactions between abiotic (reservoirs) and the three interconnected major biotic (functional) compartments. 
Further to a number of initial concepts which have been boosted in Russia from the original thoughts of V. Vemadsky, it is worth mentioning the pioneering experimental investigations promoted by C. Folsome on "ecospheres" which simulated simple marine ecosystems. These models contain one species of shrimp, defined mixtures of microalgae and bacteria, and addressed ecosystem-level features prone to be altered by toxicants such as element and energy cycling, persistence, stability and the significance of metabolic diversity [20-21-22].

A recent model, referred to as SAM (Standardized Aquatic Microcosm), has been developed as a standard practice adopted by the American Society for Testing Materials (ASTM), and is based upon 10 species of microalgae, 5 species of small animals, and (undefined) bacteria [23]. It has been used for the clarification of heavy metal and pesticide effects on ecosystem processes with the further support of appropriate modelling [15]. Another model, quite relevant although simpler, has been used recently to assess external dose of $\gamma$ and $X$ rays on ecosystem processes [18-19]. This is based on 3 micro-organisms: Tetrahymena sp. (as consumer), Euglena gracilis, (as producer), and E. coli, (as decomposer) and has demonstrated the importance of indirect effects when addressing effects at the ecosystem level.

Other quite interesting models have originated from space science where the bioregenerative properties of ecosystems (self-sustainability) are being explored for supporting human life in the purpose of future space exploration. It is of much interest to notice that this approach, although directed to a different immediate goal, by having faced problems recognised as very similar to those encountered in terrestrial environmental issues (harmful toxicant accumulation), is now promoting synergies [24].

\subsection{Suggested approach to support the environmental radioprotection needs for knowledge}

After several decades of rather classical investigations having guided the understanding of radiation effects on the environment to its current status, a significant breakthrough can only emerge from teal innovation, to be carefully lined up with the gaps in knowledge identified above. $\gamma$ irradiation through external exposure has often been the focus of the first attempts due to its much easier amenability to measurement. This is the current approach under development at the Savannah River Ecology Laboratory where batteries of mesocosms featuring artificial aquatic ecosystems are being exposed to $\gamma$ radiations for long-term investigations. The major goal aims at clarifying how and to which extent sub-cellular damages (addressed by molecular probes) may be transposed into effects at populations level [25].

However, it is argued here that the "long-term" issue in association with "low doses" drives to paying particular attention to $\alpha$ and $\beta$ radiations through internal exposure, that is to say radionuclides having penetrated the biological systems. Indeed, much concern with respect to potential harm to ecosystem health in the long-term is linked to bioaccumulation phenomena, an area which begins to attract current attention [26]. Focusing on these types of radiations is further justified from their known ability to promote larger biological effects (high RBE).

It is therefore suggested to develop a complementary experimental approach using controlled ecological models similar to those previously mentioned, but exploited for radiotoxicology purposes. At first, these are to be based on replicable, and self-organised, aquatic systems comprising essentially a few microbial biota. Higher complexities may be introduced later on, as higher trophic level biota and terrestrial ecosystem simulation. The goal consists in clarifying the chain of events which, after introducing $\alpha$ and $\beta$ emitting radionuclides into the system, may result in some alteration of valuable ecosystem functions such as integrity, stability, survivability, persistence, element cycling. The kinetic distribution of the radionuclides in the various pools (abiotic and bio-accumulated) will need to be followed up along with population indicators (simple counting, ATP content, oxidative/reductive balance, eukaryote/prokaryote ratio, etc.). The high level experimental control over ecosystem variables allows for the concomitant development of mathematical formulations which can be conceptualised/tested/upgraded at all stages.

\section{CONCLUSION}

Using controlled ecological models, in support to both experimental and mathematical simulations, presents a number of advantages prone to deriving substantial scientific information valuable to 
improving environmental radioprotection assessment. They match the identified gaps in scientific knowledge with a particular dedication to the much debated potential effects at the higher levels of organisation. They can support novel theoretical investigations by modelling, which will fruitfully seed new approaches to unravelling what is observed in the real environment. With a selection of rapidly developing biota (essentially microbial) they allow for multi-generation examination of the long-term issue, a feature practically impossible in the real environment which would require time frames in the range of decades or centuries. Ultimately, they fulfil a gap between excessive reductionism and too much holism where the still overwhelming complexity restricts to pure descriptions with limited understanding.

\section{References}

[1] ICRP, Publication 26, (Pergamon Press, Oxford, 1977).

[2] ICRP, Publication 60, (Annals of the ICRP 21, Pergamon Press, Oxford, 1991).

[3] Pentreath R.J. Radiation Protec. Dos. 75(1-4) (1998) 175-179.

[4] Larsson C.M. et al., FASSET, Technical Annex of the $5^{\text {th }}$ FP CEC Contract $n^{\circ}$ FIGE-CT-2000-00102.

[5] Whicker W. and Hinton T., "Effects of ionising radiation on terrestrial ecosystems", International Symposium on Ionizing Radiation, Stockholm, Sweden, May 20-24, B. Amiro, R. Avadhanula, G. Johansson, C.M. Larsson and M. Lüning, Eds. (1996), pp. 109-123.

[6] Sugg D.W. et al., Environ. Toxicol. Chem. 14 (1995) 6671-668.

[7] Suomela M. et al., Effect of industrial pollution on the distribution dynamics of radionuclides in boreal understorey ecosystems - EPORA (STUK, Helsinki, August 1999).

[8] Ausseil O. et al., "Effects of cadmium and zinc exposure of rainbow trout on the organism's radionuclide contamination dynamics", Third World SETAC Conference, Brighton, UK, May 21-25, (2000).

[9] Baker R.J. et al., Nature 380 (1996) 707-708.

[10] Kay J.J. et al., Futures 31(7) (1999) 721-742.

[11] Bothwell et al., Science 265 (1994) 97-100.

[12] Naeem S. et al., Nature 368 (1994) 734-737.

[13] André $M$. and Bréchignac F., Nature 371 (1994) 565.

[14] Körner C. and Amone J.A., Science 257 (1992) 1672-1675.

[15] Taub F.B., Ecological Applications 7(4) (1997) 1103-1110.

[16] Fuma S. et al., Bull. Environ. Contam. Toxicol. 65 (2000) 699-706.

[17] Fuma S. et al., Bull. Environ. Contam. Toxicol. 66 (2001) 231-238.

[18] Fuma S. et al., Int. J. Radiat. Biol. 74 (1998) 145-150.

[19] Doi M. et al., "Ecological impacts of environmental toxicants and radiation on the microbial ecosystem: a model simulation of computational microbiology", IRPA 10 Harmonization of radiation, human life and the ecosystem, Hiroshima, Japan, May 14-19,(2000) T-1-4, P-2a-115.

[20] Obenhuber D., The persistence of life measured by carbon cycling in closed ecological systems. (PhD Thesis, University of Hawaï, 1986).

[21] Shaffer J., Stability in closed ecological systems: an examination of material and energetic parameters. (PhD Thesis, University of Hawaï, 1991).

[22] Brittain A.M., The metabolic diversity, biological activity and stability of the steady-state condition in closed ecosystems. ( $\mathrm{PhD}$ Thesis, University of Hawai, 1993).

[23] ASTM, Standard practice for standardized aquatic microcosms: fresh water, (Annual Book of ASTM Standards, E1366-96, 1996), pp. 697-731.

[24] COSPAR, Life Sciences: Artificial ecosystems, (Advances in Space Research, F. Bréchignac and R. Mac Elroy, eds.), 24(3) (1999) pp. 263-413.

[25] Hinton T. et al., « Determining significant endpoints for ecological risk analysis », IAEA Specialist meeting on protection of the environment from the effects of ionising radiation, Ref. 723-J9-SP-1114.2, Vienna, Austria, August 29 - September 1, 2000).

[26] Garnier-Laplace J. and Paquet F., Bioaccumulation of radionuclides in situations of chronic exposure of ecosystems and members of the public. (ENVIRHOM Project, Report IPSN-DPRE 2000-01 / DPHD 2000-03, October 2000). 